PROCEEDINGS OF THE

AMERICAN MATHEMATICAL SOCIETY

Volume 136, Number 2, February 2008, Pages 403-407

S 0002-9939(07)08875-2

Article electronically published on October 24, 2007

\title{
ON A CONJECTURE OF BROUGHAN
}

\author{
SANTOS HERNÁNDEZ HERNÁNDEZ AND FLORIAN LUCA
}

(Communicated by Ken Ono)

\begin{abstract}
In this paper, we confirm a conjecture of Broughan, concerning the closure of the set of Fibonacci numbers in the full topology over $\mathbb{Z}$.
\end{abstract}

\section{INTRODUCTION}

Let $\mathbb{Z}$ be the ring of integers equipped with the topology $\tau$ in which the base of neighborhoods for a point $a \in \mathbb{Z}$ is given by the sets

$$
N_{a, b}=\{a+n b: n \in \mathbb{Z}\} \quad \text { for } b \in \mathbb{Z}, b \geq 1 .
$$

This topology was proposed by H. Fürstenberg in 3, and it enables a very elegant proof of the fact that the set of prime numbers is infinite (see also 1]). It is called the full topology. This topology was studied in detail in the recent paper [2], where the following conjecture was proposed.

Let $F=\left\{F_{n}\right\}_{n \geq 0}$ denote the Fibonacci sequence given by $F_{0}=0, F_{1}=1$ and

$$
F_{n+2}=F_{n+1}+F_{n} \quad \text { for all } n \geq 0 .
$$

Let $F^{-}$denote the set $\left\{(-1)^{n+1} F_{n}: n \in \mathbb{N}\right\}$.

Conjecture 1. The closure of $F \subset \mathbb{Z}$ in the topology $\tau$ is $F \cup F^{-}$.

Some numerical evidence supporting the above conjecture was given in the last section of [2]. In this note, we confirm the above conjecture.

\section{Common factors of Shifted FibonacCi numbers}

The following result is part of the main theorem in [4.

Proposition 1. Let $a$ and $b$ be arbitrary integers. Then there exist two effectively computable positive constants $C_{1}$ and $C_{2}$ depending only on $a$ and $b$ such that the inequality

$$
\operatorname{gcd}\left(F_{m}+a, F_{n}+b\right)<\exp \left(C_{1} \sqrt{n}\right)
$$

holds for all pairs of positive integers $n>m>C_{2}$ unless either both $|a|$ and $|b|$ are members of the Fibonacci sequence, or there exists $\zeta \in\{ \pm 1\}$ such that

$$
\left(5 a^{2}+4 \zeta\right)\left(5 b^{2}+4 \zeta\right)=c^{2} \quad \text { for some integer } c .
$$

Received by the editors May 2, 2006 and, in revised form, August 15, 2006.

2000 Mathematics Subject Classification. Primary 11B36; Secondary 11J68.

(C)2007 American Mathematical Society 


\section{The Proof of Conjecture 1}

Throughout this section we shall denote by $\partial F$ the set of cluster points of $F$ and by $\bar{F}$ the closure of $F$ in the full topology of $\mathbb{Z}$. It is well known that $\bar{F}=F \cup \partial F$.

We proceed in three steps. We first show that if $a$ is a cluster point of $F$, then $|a| \in F$. Secondly, we show that integers of the form $-F_{\ell}$ for some odd integer $\ell>1$ are not in the closure of $F$. Finally, we show that integers in $F \cup F^{-}$are indeed cluster points of $F$.

Step 1. If $a$ is a cluster point of $F$, then $|a| \in F$. Assume that $a \in \partial F$. Thus, $\left(N_{a, b}-\{a\}\right) \cap F \neq \emptyset$ for any positive integer $b$. We choose $b=F_{m}$, where $m$ is a large positive integer that is not a multiple of 3 . It is well known that

$$
F_{m} \mid F_{n} \text { if and only if } m \mid n \text {. }
$$

Since $2=F_{3}$, we get that $2 \mid F_{n}$ if and only if $3 \mid n$. Hence, $F_{m}$ is odd. We deduce that for each such $m$ there exists a nonnegative integer $n$ such that $F_{n} \equiv a$ $\left(\bmod F_{m}\right)$. We next notice that the sequence $\left\{F_{n}\right\}_{n \geq 0}$ is periodic modulo $F_{m}$ with period $4 m$. To see why, let us first recall the well-known formulas

$$
2 F_{s+t}=F_{s} L_{t}+F_{t} L_{s} \quad \text { and } \quad L_{4 u}=5 F_{2 u}^{2}+2,
$$

where $\left\{L_{n}\right\}_{n \geq 0}$ denotes the Lucas sequence given by $L_{0}=2, L_{1}=1$ and $L_{n+2}=$ $L_{n+1}+L_{n}$ for all $n \geq 0$. Taking $t=4 m$ in the first relation (6) above we get

$$
2 F_{s+4 m}=F_{s} L_{4 m}+F_{4 m} L_{s} .
$$

Furthermore, taking $u=m$ in the second relation (6) and inserting the resulting relation in (7) we get

$$
2 F_{s+4 m}=2 F_{s}+\left(F_{4 m} L_{s}+5 F_{s} F_{2 m}^{2}\right) .
$$

From (5), we have that $F_{m} \mid F_{2 m}$ and $F_{m} \mid F_{4 m}$; thus $F_{m} \mid F_{4 m} L_{s}+5 F_{s} F_{2 m}^{2}$. Hence, reducing the relation (8) modulo $F_{m}$ we get

$$
2 F_{s+4 m} \equiv 2 F_{s} \quad\left(\bmod F_{m}\right)
$$

and since $F_{m}$ is odd we get that $F_{s+4 m} \equiv F_{s}\left(\bmod F_{m}\right)$. Thus, $\left\{F_{n}\right\}_{n \geq 0}$ is periodic with period $4 m$ modulo $F_{m}$.

Since there exists a nonnegative integer $n$ such that $F_{n} \equiv a\left(\bmod F_{m}\right)$, it follows from the above arguments that such an integer $n$ can be found in the interval $[4 m, 8 m)$. Thus, $n>m>n / 8$ and

$$
\operatorname{gcd}\left(F_{m}+0, F_{n}-a\right)=F_{m} \geq \frac{\alpha^{m}}{3}>\frac{\alpha^{n / 8}}{3}
$$

where $\alpha=(1+\sqrt{5}) / 2$. Here, we used the fact that the inequality $F_{k} \geq \alpha^{k} / 3$ holds for all positive integers $k$, the proof of which is an easy exercise using induction. From Proposition 1, we get that either $|a|$ is a member of the Fibonacci sequence, or there exist integers $\zeta \in\{ \pm 1\}$ and $c$ such that

$$
4 \zeta\left(5 a^{2}+4 \zeta\right)=c^{2}
$$

or there exist positive constants $C_{1}$ and $C_{2}$ depending on $a$ such that if $n>8 C_{2}$, then $m>n / 8>C_{2}$ and

$$
\operatorname{gcd}\left(F_{m}, F_{n}-a\right)<\exp \left(C_{1} \sqrt{n}\right) .
$$

The first alternative gives us the desired conclusion. For the second alternative, note that unless $a=0$ (which is in $F$ ), we have that $5 a^{2}+4 \zeta>0$. Hence, equation 
(10) forces $\zeta=1, c=2 c_{1}$ to be even, and $5 a^{2}+4=c_{1}^{2}$. Thus, $c_{1}^{2}-5 a^{2}=4$. However, it is well known (see, for example, Theorems 27.1 and 27.2 on page 338 of [5]) and follows easily from the continued fraction expansion of the golden section $(1+\sqrt{5}) / 2$, that if $(x, y)$ is an integer solution of the Pell-type equation $x^{2}-5 y^{2}= \pm 4$, then there exists a nonnegative integer $t$ such that $x= \pm L_{t}$ and $y= \pm F_{t}$. Hence, $|a| \in F$ in this case also. Finally, the third alternative leads to the conclusion that both inequalities (11) and (9) hold, therefore that

$$
\exp \left(C_{1} \sqrt{n}\right)>\frac{\alpha^{n / 8}}{3} \quad \text { provided that } n>8 C_{2}
$$

which leads to the conclusion that $n<C_{3}$ is bounded. This is however impossible since $n \geq 4 m$ and $m$ can be chosen to be arbitrarily large. This concludes the proof of the fact that if $a \in \partial F$, then $|a| \in F$.

Step 2. If $a=-F_{\ell}$ where $\ell>1$ is odd, then $a \notin \partial F$. In order to show this, it is enough to display for each such $\ell$ a positive integer $b$ such that the congruence $F_{n} \equiv-F_{\ell}(\bmod b)$ has no nonnegative integer solution $n$.

We now fix the number $\ell$ and first show that if $16 \mid b$, then $n$ cannot be odd. Indeed, assume that $n$ is odd. Since the Fibonacci sequence is periodic modulo $b$, there must exist infinitely many positive integers $n$ such that $F_{n} \equiv-F_{\ell}(\bmod b)$, and we choose one such that $n>\ell$. Let $\delta \in\{ \pm 1\}$ be such that $n+\delta \ell \equiv 2(\bmod 4)$. Note that $n \equiv \delta \ell(\bmod 4)$. The formula

$$
F_{n}+F_{\ell}=F_{(n+\delta \ell) / 2} L_{(n-\delta \ell) / 2} \quad \text { whenever } \quad n \equiv \delta \ell \quad(\bmod 4)
$$

can be easily checked, for example, by a direct calculation using the known formulas

$$
F_{n}=\frac{\alpha^{n}-\beta^{n}}{\alpha-\beta} \quad \text { and } \quad L_{n}=\alpha^{n}+\beta^{n}, \quad \text { where } \quad \beta=-\alpha^{-1} .
$$

The sequence $\left\{F_{n}\right\}_{n \geq 0}$ is periodic modulo 4 with period 6 and the sequence $\left\{L_{n}\right\}_{n \geq 0}$ is periodic modulo 8 with period 12. By looking at the first six values of the Fibonacci sequence and at the first twelve values of the Lucas sequence one notices that $F_{k}$ is never a multiple of 4 if $k$ is odd and that $L_{k}$ is never a multiple of 8 . Since the index $(n+\delta \ell) / 2$ of the Fibonacci number appearing in the right-hand side of formula (12) is odd, we conclude from relation (12) that $F_{n}+F_{\ell}$ is never a multiple of 16. Hence, it is impossible that $b \mid F_{n}+F_{\ell}$ if $16 \mid b$ and $n$ is odd.

From now on, we suppose that $n$ is even. We take $b=16 F_{m}$, where again $m$ is a large odd positive integer that is not a multiple of 3 . Since $F_{n} \equiv a\left(\bmod F_{m}\right)$ holds with some even $n$, it follows, by the fact that $\left\{F_{n}\right\}_{n \geq 0}$ is periodic modulo $F_{m}$ with period $4 m$, that we may assume that $n$ is still even in $[4 m, 8 m)$.

We now follow again the method from $[4]$. Let $\mathbb{K}=\mathbb{Q}[\sqrt{5}]$. In $\mathbb{K}$, we have that

$$
F_{m}=\frac{1}{\sqrt{5}}\left(\alpha^{m}-\beta^{m}\right)=\frac{1}{\sqrt{5}}\left(\alpha^{m}-\left(-\alpha^{-1}\right)^{m}\right)=\frac{\alpha^{-m}}{\sqrt{5}}\left(\alpha^{2 m}+1\right) .
$$

On the other hand,

$$
\begin{aligned}
F_{n}-a & =\frac{1}{\sqrt{5}}\left(\alpha^{n}-\beta^{n}\right)-a=\frac{1}{\sqrt{5}}\left(\alpha^{n}-\left(-\alpha^{-1}\right)^{n}\right)-a \\
& =\frac{\alpha^{-n}}{\sqrt{5}}\left(\alpha^{2 n}-\sqrt{5} a \alpha^{n}-1\right)=\frac{\alpha^{-n}}{\sqrt{5}}\left(\alpha^{n}-z_{1}\right)\left(\alpha^{n}-z_{2}\right),
\end{aligned}
$$


where

$$
z_{1,2}=\frac{\sqrt{5} a \pm \sqrt{5 a^{2}+4}}{2} .
$$

Note that since $a=-F_{\ell}$ and $\ell$ is odd, we have that $L_{\ell}^{2}-5 F_{\ell}^{2}=(-1)^{\ell} 4=-4$; therefore $5 a^{2}+4=L_{\ell}^{2}+8$. Note that $5 a^{2}+4$ is not a multiple of 5 , and it is not a square since otherwise with $x=L_{\ell}$, we would get that $x^{2}+8=y^{2}$ for some positive integer $y$, or $8=(y-x)(y+x)$. The only solution in positive integers $x, y$ of the above equation is $x=1, y=3$, but this is not convenient since for us $\ell>1$; therefore $x=L_{\ell}>1$. Thus, we deduce that $5 a^{2}+4=d y^{2}$, where $d>1$ is a square-free integer that is not a multiple of 5 .

Let $\mathbb{L}=\mathbb{K}\left[z_{1}\right]$. The above remarks show that $[\mathbb{L}: \mathbb{Q}]=4$. Since $F_{m} \mid F_{n}-a$, it follows that in $\mathbb{L}$ we have that $\left(\alpha^{2 m}+1\right)$ divides $\left(\alpha^{n}-z_{1}\right)\left(\alpha^{n}-z_{2}\right)$. Here, for an algebraic integer $\gamma$ in $\mathbb{L}$ we write $(\gamma)$ for the principal ideal (in the ring of algebraic integers $\mathcal{O}_{\mathbb{L}}$ of $\left.\mathbb{L}\right)$ generated by $\gamma$. Let $I_{i}=\operatorname{gcd}\left(\left(\alpha^{2 m}+1\right),\left(\alpha^{n}-z_{i}\right)\right)$ for $i=1,2$. Then $\left(F_{m}\right) \mid I_{1} I_{2}$ in $\mathbb{L}$.

We shall next bound the norm of $I_{i}$ in $\mathbb{L}$ for $i=1$, 2. Let

$$
\mathcal{S}=\left\{\lambda n+2 \mu m: \lambda, \mu \in\left\{1, \ldots,\left\lfloor m^{1 / 2}\right\rfloor\right\} .\right.
$$

If $s=\lambda n+2 \mu m$, then $1 \leq s \leq(n+2 m) m^{1 / 2}<10 m^{3 / 2}$. Since there are $\left(\left\lfloor m^{1 / 2}\right\rfloor\right)^{2}$ pairs of positive integers $(\lambda, \mu)$ with $\lambda, \mu \in\left\{1, \ldots,\left\lfloor m^{1 / 2}\right\rfloor\right\}$, it follows, by the Pigeon-Hole Principle, that there exist two distinct pairs $\left(\lambda_{1}, \mu_{1}\right) \neq\left(\lambda_{2}, \mu_{2}\right)$ such that

$$
\left|\left(\lambda_{1}-\lambda_{2}\right) n+2\left(\mu_{1}-\mu_{2}\right) m\right|<\frac{10 m^{3 / 2}}{\left\lfloor m^{1 / 2}\right\rfloor^{2}-1}<11 m^{1 / 2} \quad \text { for } m \text { large enough. }
$$

Writing $x=\lambda_{1}-\lambda_{2}$ and $y=\mu_{1}-\mu_{2}$, we get that $(x, y) \neq(0,0)$, that $x, y \in$ $\left(-m^{1 / 2}, m^{1 / 2}\right)$ and that if we write $s=n x+2 m y$, then $|s|<11 m^{1 / 2}$. Note now that

$$
\alpha^{2 m} \equiv-1 \quad\left(\bmod I_{i}\right) \quad \text { and } \quad \alpha^{n} \equiv z_{i} \quad\left(\bmod I_{i}\right) .
$$

Raising the first congruence to the power $y$ and the second to the power $x$ (notice that such operations are justified even if $x$ and $y$ are negative since $\alpha$ is a unit in $\mathbb{K}$, therefore also in $\mathbb{L}$ ), and multiplying the resulting congruences we get

$$
\alpha^{s} \equiv(-1)^{y} z_{i}^{x} \quad\left(\bmod I_{i}\right) .
$$

Thus, $I_{i}$ divides $\left(\alpha^{s}-(-1)^{y} z_{i}^{x}\right)$. Note that this last ideal is not zero. Indeed, for if not, then we would get that $\alpha^{2 s}=z_{i}^{2 x}$. By replacing the pair $(x, y)$ by the pair $(-x,-y)$, we may assume that $x \geq 0$. Since it is easy to see that $z_{i}^{2} \in \mathbb{Q}[\sqrt{5 d}]$ and it is not a rational number, it follows that $z_{i}^{2 x} \in \mathbb{Q}[\sqrt{5 d}]$ also and it is not a rational number whenever $x>0$, while $\alpha^{2 s} \in \mathbb{K}$. Thus, the only possibility that $z_{i}^{2 x}=\alpha^{2 s}$ is $x=s=0$, and since $s=n x+2 m y$, we also get that $y=0$, which contradicts the fact that $(x, y) \neq(0,0)$. Hence, $I_{i}$ divides the nonzero ideal $\left(\alpha^{s}-(-1)^{y} z_{i}^{x}\right)$. Thus,

$$
\left(F_{m}\right)\left|I_{1} I_{2}\right|\left(\alpha^{s}-(-1)^{y} z_{1}^{x}\right)\left(\alpha^{s}-(-1)^{y} z_{2}^{x}\right),
$$

and the ideal appearing in the right-hand side above is not zero. Taking norms in $\mathbb{L}$, we get that

$$
\begin{aligned}
F_{m}^{4} & =N_{\mathbb{L}}\left(F_{m}\right) \leq N_{\mathbb{L}}\left(\left(\alpha^{s}-(-1)^{y} z_{1}^{x}\right)\left(\alpha^{s}-(-1)^{y} z_{2}^{x}\right)\right) \\
& <\left(2^{s}+\left(5 a^{2}+4\right)^{x / 2}\right)^{8}<2^{8}\left(5 a^{2}+4\right)^{\max \{8 s, 4 x\}},
\end{aligned}
$$


where in the above inequalities we have used the fact that $\alpha$ and its conjugate are less than 2 in absolute value and that $z_{1}, z_{2}$ and all their conjugates (in $\mathbb{L}$ ) are less than $\sqrt{5 a^{2}+4}$ in absolute values (see (16)). Since $|s|<11 m^{1 / 2}$ and $x<m^{1 / 2}$, we get that

$$
\left(\frac{\alpha^{m}}{3}\right)^{4} \leq F_{m}^{4} \leq 2^{8}\left(5 a^{2}+2\right)^{88 m^{1 / 2}},
$$

which leads to the conclusion that $m$ is bounded, which is of course a contradiction. This shows that indeed if $a=-F_{\ell}$ and $\ell$ is odd, then $a \notin \bar{F}$.

Step 3. The elements of $F^{-}$are cluster points of $F$. Since the Fibonacci sequence $\left\{F_{n}\right\}_{n \geq 0}$ is periodic modulo $b$ for any positive integer $b$, it follows that if we write $e_{b}$ for this period, then $F_{n+t e_{b}} \equiv F_{n}(\bmod b)$ for any $t \geq 0$. Hence, $N_{F_{n}, b}$ contains infinitely many members of $F$. Finally, it is a well-known fact that we may use recurrence (2) to define $F_{n}$ for negative values of $n$, and in this case the formula for $F_{n}$ appearing in (13) holds for negative values of $n$ also. Furthermore, using that formula (or by induction using the recurrence (2)) one checks that $(-1)^{n+1} F_{n}=$ $F_{-n}$. Since this extended sequence $\left\{F_{n}\right\}_{n \in \mathbb{Z}}$ remains periodic modulo $b$ with the same period $e_{b}$, we get that $F_{-n+t e_{b}} \equiv F_{-n}(\bmod b)$ for all $n \geq 0$ and all integers $t$. Taking $t$ to be a sufficiently large positive integer (i.e., such that $-n+t e_{b}>0$ ), we see that $N_{F_{-n}, b}$ contains members of $F$; therefore $F_{-n} \in \partial F$.

Recall that $\bar{F}=F \cup \partial F$. Step 3 shows that $F \cup F^{-} \subseteq \bar{F}$. Steps 1 and 2 show that $\bar{F} \subseteq F \cup F^{-}$. Thus, $\bar{F}=F \cup F^{-}$.

\section{ACKNOWLEDGMENTS}

The authors thank Luis Adrian Martínez Pérez for drawing their attention to Broughan's paper 22. They also thank the anonymous referee for comments which improved the quality of this paper. This work was done in April of 2006, while the second author was in residence at the Centre de Recherches Mathématiques of the Université de Montréal for the thematic year Analysis and Number Theory. This author thanks the organizers for the opportunity of participating in this program. The first author was supported by the Postdoctoral Fellowship MECESUP PUC0103. The second author was also supported in part by Grants SEP-CONACyT 46755, PAPIIT IN104505 and a Guggenheim Fellowship.

\section{REFERENCES}

[1] M. Aigner and G. M. Ziegler, Proofs from The Book, Springer-Verlag, 1998.

[2] K. A. Broughan, 'Adic Topologies for the Rational Integers' Canad. J. Math. 55 (2003), 711-723. MR1994070 (2004i:11005)

[3] H. Fürstenberg, 'On the infinitude of primes', Amer. Math. Monthly 62 (1955), 353. MR0068566 (16:904e)

[4] S. Hernández and F. Luca, 'Common Factors of Shifted Fibonacci Numbers', Per. Math. Hungarica 47 (2003), 95-110. MR2024976 (2004h:11017)

[5] T. Koshy, Fibonacci and Lucas numbers with applications, Pure and Applied Mathematics (New York). Wiley-Interscience, New York, 2001. MR1855020 (2002f:11015)

Facultad de Matemáticas, Pontificia Universidad Católica de Chile, Vicuña Mackenna 4860, Santiago, Chile

E-mail address: shernand@mat.puc.cl

Instituto de Matemáticas, Universidad Nacional Autónoma de México, C.P. 58089, Morelia, MichoacÁn, México

E-mail address: fluca@matmor.unam.mx 